\title{
The genotype analysis and prenatal genetic diagnosis among 244 pedigrees with methylmalonic aciduria in China
}

\author{
Shuang Hu \\ First Affiliated Hospital of Zhengzhou University \\ Lina Liu \\ First Affiliated Hospital of Zhengzhou University \\ Zhihui Jiao \\ First Affiliated Hospital of Zhengzhou University \\ Xiangdong Kong ( $\square$ kongxd@263.net ) \\ First Affiliated Hospital of Zhengzhou University
}

\section{Research Article}

Keywords: methylmalonic aciduria, MMACHC, MUT, variant, prenatal genetic diagnosis

Posted Date: February 24th, 2021

DOI: https://doi.org/10.21203/rs.3.rs-249909/v1

License: (-) This work is licensed under a Creative Commons Attribution 4.0 International License. Read Full License 


\section{Abstract}

To investigate the phenotypes, biochemical features and genotypes for 244 pedigrees with methylmalonic aciduria (MMA) in China, and to perform the prenatal genetic diagnosis by chorionic villus for these pedigrees. Gene analyses were performed for 244 pedigrees. There are 130 families, chorionic villus sampling was performed on the pregnant women to conduct the prenatal diagnosis. Among 244 patients, 168 (68.9\%) cases were combined methylmalonic aciduria and homocystinuria, 76 (31.1\%) cases were isolated methymalonic aciduria. All the patients were diagnosed with MMA by their clinical manifestation, elevated blood propionylcarnitine, propionylcarnitine to acetylcarnitine ratio, and/or urine/blood methylmalonic acid with or without hyperhomocysteinemia. MMACHC, MUT, SUCLG1 and LMBRD1 gene variants were found in 236 (96.7\%) pedigrees included 6 probands with only one heterozygous variant out of 244 cases. For the 130 pedigrees who received a prenatal diagnosis, 22 foetuses were normal, 69 foetuses were carriers of heterozygous variants, and the remaining 39 foetuses harboured compound heterozygous variants or homozygous variants. The follow-up results were consistent with the prenatal diagnosis. The present study indicates genetic heterogeneity in MMA patients. Genetic analysis is a convenient method for prenatal diagnosis that will aid in avoiding the delivery of MMA patients.

\section{Background}

Methylmalonic acidaemia or aciduria (MMA) is a seriously fatal inborn error of organic acid metabolism [1, 2], it is a kind of disease caused by deficiency of methylmalonyl coenzyme A mutase (MCM) or intracellular cobalamin (cbl) metabolism [3]. To date, it has been reported that more than 10 pathogenicity genes could cause inherited MMA, most of which are autosomal recessive $[4,5]$. The defects of these pathogenic genes like MMACHC, MUT, MMAA, MMAB, MMADHC, LMBRD1, result in methylcobalamin and adenosylcobalamin dysfunction, leading to the abnormal high concention of blood and urine methylmalonic acid, and this is a biochemical characterization of the disease [6,7]. There are two main forms of MMA classified as isolated MMA or combined methylmalonic aciduria and heperhomocystinuria on the base of the patients' biochemical features. The former consists of 4 subtypes, including MUT defect, Cobalamin A(cblA,MMAA gene), cblB $(M M A B)$ and cbID variant 2(MMADHC) defect [8-10], the latter consists of five subtypes, including $\operatorname{cbIC}(M M A C H C), \operatorname{cblD}(M M A D H C), \operatorname{cblF}(\angle M B R D 1), \operatorname{cblJ}(A B C D 4)$ and $\operatorname{cblX}(H C F C 1)$ deficiencies [11-13]. There are some other genes that can cause abnormal methylmalonic acid in blood and urine, but the name of the disease is not methylmalonic acid. For example, mitochondrial DNA depletion sysdrome (MDS) caused by SUCLG1 or SUCLA2 gene [14], combined malonic and methylmalonic aciduria due to ACSF3 variants [15], MCEE gene defect result in MethylmalonylCoA Epimerase Deficiency [16].

In China, combined methylmalonic acidaemia and homocystinuria is the most common type in the affected patients, accounting for nearly $70 \%$ of all MMA cases [17]. Among the subtypes, the most common type is the cblC defect (MIM 277400) caused by MMACHC gene variants [18], which is located in chromosome region 1p34.1, the gene contains five exons and encodes a protein of 282 amino acid [19]. For the isolated MMA, MUT gene is the most common one which is located in chromosome region 6p12.3 and contains 13 exons and encodes a 750 amino acid protein [20], other disease-causing genes including MMAA(MIM 251100), $M M A B(\mathrm{MIM} 251110), M M A D H C(M I M) 277410)$, whose incidence rate are all very low.

The common manefitations of isolated methylmalonic aciduria are lethargy, vomiting, hepatomegaly, hypotonia, metabolic acidemia and in many, hyperammonemic encephalopathy. Some of them can exhibit feeding problems (typically anorexia), failure to thrive, and developmental delay. Most of mut patients are at a risk of develop end stage renal failure[21-23]. However, the clinical presentation of combined methlymalonic aciduria and homosystinuria includes poor feeding, dehydration and lethargy, failure to thrive and developmental delay for infants. cblC patients are prone to developing an atypical hemolytic uremic syndrome, secondary to thrombotic microangiopathy. Some patients will suffer from progressive encephalopathy or pulmonary arterial hepertension with age. Patients can present in any decade of life with neurological impairments, which are usually irreversible. [6, 24, 25]. Most affected patients present early in their life with poor prognosis, if without timely diagnosis and intervention they usually died in their first year old age $[25,26]$. Nevertheless, these years thanks to expanded newborn screening, particularly gas chromatography-mass spectrometry (GC-MS), more and more MMA patients have benefited due to an early diagnosis and better prognosis [27]. Even with early screening and proper treatment, the long-term complications like kidney disease and neurological disorder would make the probands' life quality very low, and the families of these patients suffer an enormous economic burden. Therefore, to avoid the birth of children with MMA by prenatal diagnosis in China is an effective measure to reduce the economic 
pressure. Some institutions measured the activity of methylmalonyl CoA mutase in amniotic fluid, amniotic fluid cells and chorionic cells, but others quantified cbl metabolites in amniotic fluid cells to conduct prenatal diagnosis [28, 29]. Here, our study performs the prenatal genetic diagnosis by chorionic villus.

To get further knowledge of this disease, we recruited 244 pedigrees with methylmalonic acidaemia who visited the Genetic Counseling Clinic of the First Affiliated Hospital of Zhengzhou University between January 2014 and July 2018. The MMA pathogenicity related genes in the probands and their parents were analysed by Sanger sequencing or Next Generation Sequencing (NGS). One hundred and thirty pregnant mothers in this cohort of Chinese MMA pedigrees choose to perform the prenatal genetic diagnosis. The primary aim of our study was to provide genetic-based prenatal diagnosis of MMA in China.

This study was approved by the Medical Ethics Committee of the First Affiliated Hospital of Zhengzhou University. All analysed samples were obtained after signed informed consent was provided.

\section{Methods}

\section{Pedigrees and MMA diagnosis}

Our study collected 244 pedigrees including couples who had a child birth history of MMA. To diagnose the patients, we should perform complementation analysis on the clinical presentation, positive methylmalonic acid results in blood and urine or elevated blood propionylcarnitine and propionylcarnitine to acetylcarnitine ratio by gas chromatography-mass spectrometry (GC-MS, QP2010, Schimadzu, Japan) and. tandem mass spectrometry (MS-MS, AB Sciex, API 4000, California, United States ), respectively. The plasma or urine homocysteinemia was detected by Fluorescence polarization immunoassay (FPLA) to distinguish isolated or combined MMA. The patients were enrolled from the Genetic Counseling Clinic of the First Affiliated Hospital of Zhengzhou University between January 2014 and July 2018. No probands were consanguineous marriages. One hundred healthy individuals without MMA served as controls.

\section{DNA extraction}

Peripheral blood samples were collected in EDTA tubes from 200 probands and 244 couples. For families with a deceased patient, specimens were merely collected from their parents. The transabdominal chorionic villi sampling were performed at 11 to 14 weeks of gestation. Genomic DNA was extracted from each sample using a DNA extraction kit (Omega blood/tissue DNA kit, Georgia, United States) following the manufacturer's protocal.

\section{Sequencing and the detection of deletions/duplications in the MMACHC/MUT genes}

The polymerase chain reaction (PCR) primers were designed in line with previously published data [30]. The coding exons and splicing regions of the MMACHC and MUT gene were amplified by PCR for pedigree 1 to 155, and subsequently, the PCR products were sequenced bi-directionally using an ABI 3130-xl gene analyser (Life Technologies, Carlsbad, CA, United States). In order to identify these nucleotides, all the sequences were aligned and inspected using a reference sequence from Ensemble (NM_015506) (http://asia.ensembl.org/). For pedigree 156 to pedigree 244, Next Generation Sequencing (NGS) was applied to test the DNA through lon Torrent sequencing platform by using the common inherited metabolic disorder panel, which contains all the 22 pathogenic genes related to methylmalonic aciduria and propionic acidemia, including the ABCD4 ACSF3 ALDH6A1 GIF LMBRD1 MCEE MMAA MMAB MMACHC MMADHC MOCS1 MOCS2 MUT SUCLA2 SUCLG1 PCCA PCCB HCFC1 CBLIF AMN CUBN TCN2 gene. Ion Torrent data analysis uses Ion Torrent Suite v3.0 software. To avoid false positives, the suspicious pathogenic variants were verified by Sanger sequencing.

The real-time quantitative PCR (Q-PCR) technology was used to test deletions /duplications in the MMACHC/MUT genes for pedigrees with only one or no pathogenic variant. The exon deletion was confirmed by microarray-based comparative genomic hybridization [31].

\section{Genotype analysis}


These variants were identified by searching the HGMD database (http://www.hgmd.cf.ac.uk/ac/index.php.), Single nucleotide polymorphisms (SNPs) were further excluded by querying known disease databases, like 1000 Genomes Data (http://www.1000genomes.org/) and dbSNP (http://www.ncbi.nlm.nih.gov/), and the ExAC consortium (http://exac.broadinstitue.org/ ). The novel variants were named by using the international gene mutation nomenclature system (http://www.HGVS.org/varnomen). To predict the possible pathogenicity of the variations, we calculated by using Mutation taster, Polymorphism phenotyping (PolyPhen) and SIFT.

\section{Prenatal diagnosis}

When the genotype of the probands and their parents were confirmed, prenatal diagnosis was conducted by chorionic villus sampling of 130 pregnant women. For the sake of mathers' contamination, a PowerPlex 16 HS System kit (Promega, Madison, WI, USA) was used. All the results were analysed using ABI 3130xl and GeneMapper v3.2 software.

\section{Follow-up}

Umbilical cord blood was obtained for fetal genetic diagnosis.

\section{Results}

\section{Clinical and biochemical features}

As a whole, the patients came from different provinces in China, most of them came from Henan and Shandong Province. 224 patients of the 244 (83.6\%) pedigrees were recruited by after being suspected of MMA, 20 probands (16.3\%) were identified via newborn screening. The diagnosis of all the patients was established based on the clinical presentation, positive methylmalonic acid results in urine/blood or elevated blood propionylcarnitine, propionylcarnitine to acetylcarnitine ratio. At the same time, the plasma or urine homocysteinemia was detected by Fluorescence polarization immunoassay (FPLA) to distinguish isolated or combined MMA.

Their main clinical manifestations includes intermittent matabolic decompensation like metabolic acidosis, severe ketoacidosis, hyperammonemia, and lethargy, vomitting, hypotonia, failure to thrive, feeding difficulties and respiratory distress. Some patients have compliacations in neuro system, hematological system, liver or cardiovascular system.

In total, 168 affected patients are combined MMA, the other 76 affected people are isolated MMA. And 230 out of 244 pedigrees had homozygous or compound heterozygous variants, 6 of them had only one variant allele of the genes we detected, for the left 8 pedigrees no related variants were found.

\section{Mma Related Gene Variant Spectrum}

For all 244 probands who received gene analysis in our study, we found 4 pathogenic related genes, including $M M A C H C, M U T$, LMBRD1 and SUCLG1 (Table 1). The detailed allele variants of 236 pedigrees included 6 probands with only one heterozygous variant in our study is presented in Table 2 (MMACHC), Table 3 (MUT) and Table 4 (SUCLG1 and LMBRD1). Among these variants, the most prevalent variant in $M M A C H C$ affected patients was c.609G > A (p.Trp203Ter) (48.28\%), followed by c.658_660delAAG (p.Lys220del) (13.17\%) and c.567dupT (p.lle190Tyrfs*13) (7.84\%). The most prevalent variant in MUT affected patients was c.729_730insTT (p.Asp244Leufs*39) (16.78\%), closely followed by c.1106G > A (p.Arg369His) (7.69\%), c.914T > C (p.Leu305Ser) (6.99\%) and c.323G > A (p.Arg108His) (6.29\%). None of the variants described above were identified among 100 healthy control subjects. 
Table 1

The genotype of 244 pedigrees affected by MMA

\begin{tabular}{|c|c|c|c|}
\hline gene name & case number & known variants & new variants \\
\hline$M M A C H C$ & $158+3^{\star 1}$ & 34 & 2 \\
\hline MUT & $70+3^{\star 2}$ & 60 & 19 \\
\hline LMBRD1 & 1 & 1 & \\
\hline SUCLG1 & 1 & 1 & \\
\hline negative & 8 & & \\
\hline \multicolumn{4}{|c|}{$\begin{array}{l}\text { *1 means } 158 \text { pedigrees have } 2 \text { allele variants, whileas } 3 \text { pedigrees have only found one heterozygous } M M A C H C \text { gene variant. } \\
\text { * } 2 \text { means } 70 \text { pedigrees have } 2 \text { allele variants in MUT gene, whileas } 3 \text { pedigrees have only found } 1 \text { heterozygous MUT gene } \\
\text { variant. }\end{array}$} \\
\hline
\end{tabular}


Table 2

MMACHC gene variants for $158+3$ pedigrees

\begin{tabular}{|c|c|c|c|c|c|}
\hline cDNA change & amino acid change & exon & effect & frequency & percentage \\
\hline c. $1 A>G$ & p.Met1Val & 1 & misense variant & 1 & $0.31 \%$ \\
\hline c. $80 A>G$ & p.GIn27Arg & 1 & misense variant & 16 & $5.02 \%$ \\
\hline $\mathrm{c} .81+1 \mathrm{G} \square \mathrm{A}$ & & intron1 & splicing variant & 1 & $0.31 \%$ \\
\hline c. $89 \mathrm{G}>\mathrm{A}$ & p.Trp30Ter & 2 & nonsense varaint & 1 & $0.31 \%$ \\
\hline c. $217 \mathrm{C}>\mathrm{T}$ & p.Arg73Ter & 2 & nonsense varaint & 12 & $3.76 \%$ \\
\hline c.270_271insA & p.Arg91Lysfs*14 & 2 & frameshift variant & 2 & $0.63 \%$ \\
\hline c. $315 C>G$ & p.Tyr105Ter & 3 & nonsense varaint & 5 & $1.57 \%$ \\
\hline c. $331 \mathrm{C}>\mathrm{T}$ & p.Arg111Ter & 3 & nonsense varaint & 2 & $0.63 \%$ \\
\hline c.328_331delAACC & p.Asn110Aspfs*13 & 3 & frameshift variant & 1 & $0.31 \%$ \\
\hline c. $365 \mathrm{~A}>\mathrm{T}$ & p.His122Leu & 3 & misense variant & 1 & $0.31 \%$ \\
\hline c. $394 \mathrm{C}>\mathrm{T}$ & p.Arg132Ter & 3 & nonsense varaint & 8 & $2.51 \%$ \\
\hline c.415_416delinsTA & p.Pro139Ter & 3 & nonsense varaint & 1 & $0.31 \%$ \\
\hline c.440_441delGT & p.Cys149Hisfs*32 & 4 & frameshift variant & 1 & $0.31 \%$ \\
\hline c. $440 \mathrm{G}>\mathrm{A}$ & p.Gly147Asp & 4 & misense variant & 2 & $0.63 \%$ \\
\hline c.445_446insA & p.Cys149Ter & 4 & frameshift variant & 2 & $0.63 \%$ \\
\hline c.445_446delTG & p.Cys149Hisfs*32 & 4 & frameshift variant & 7 & $2.19 \%$ \\
\hline c.445_446delT & p.Cys149Alafs*15 & 4 & frameshift variant & 1 & $0.31 \%$ \\
\hline c. $463 G>C$ & p.Gly155Arg & 4 & misense variant & 1 & $0.31 \%$ \\
\hline c.463_465delGGG & p.Gly155del & 4 & deletion variant & 1 & $0.31 \%$ \\
\hline c.465_467delGGG & p.Gly155del & 4 & deletion variant & 1 & $0.31 \%$ \\
\hline c. $467 \mathrm{G}>\mathrm{A}$ & p.Gly156Ala & 4 & misense variant & 2 & $0.63 \%$ \\
\hline c. $481 \mathrm{C}>\mathrm{T}$ & p.Arg161Ter & 4 & nonsense varaint & 5 & $1.57 \%$ \\
\hline c. $482 \mathrm{G} \square \mathrm{A}$ & p.Arg161GIn & 4 & misense variant & 13 & $4.08 \%$ \\
\hline c. $565 \mathrm{C}>\mathrm{T}$ & p.Arg189Cys & 4 & misense variant & 1 & $0.31 \%$ \\
\hline c.567_568 ins T & p.lle190Tyrfs*13 & 4 & frameshift variant & 25 & $7.84 \%$ \\
\hline c. $599 \mathrm{G}>\mathrm{A}$ & p.Trp200Ter & 4 & nonsense varaint & 1 & $0.31 \%$ \\
\hline c.609GロA & p.Trp203Ter & 4 & nonsense varaint & 154 & $48.28 \%$ \\
\hline c. $615 \mathrm{C}>\mathrm{A}$ & p.Tyr205Ter & 4 & nonsense varaint & 2 & $0.63 \%$ \\
\hline c. $617 \mathrm{G}>\mathrm{A}$ & p.Arg206Gln & 4 & misense variant & 1 & $0.31 \%$ \\
\hline c.626dupT & p.Thr210Aspfs*35 & 4 & frameshift variant & 2 & $0.63 \%$ \\
\hline c.637G口T & p.Glu213Ter & 4 & nonsense varaint & 2 & $0.63 \%$ \\
\hline c.658_660 delAAG & p.Lys220del & 4 & deletion variant & 42 & $13.17 \%$ \\
\hline c. $666 \mathrm{C}>\mathrm{A}$ & p.Tyr222Ter & 4 & nonsense varaint & 1 & $0.31 \%$ \\
\hline c. $683 \mathrm{C}>\mathrm{T}$ & p.Ala228Val & 4 & misense variant & 1 & $0.31 \%$ \\
\hline
\end{tabular}


Table 3

MUT gene variants for $70+3$ pedigrees

\begin{tabular}{|c|c|c|c|c|c|}
\hline cDNA change & amino acid change & exon & effect & frequency & percentage \\
\hline c. $-39-2 A>G$ & & 5'UTR & 5'UTR variant & 1 & $0.70 \%$ \\
\hline c. $3 \mathrm{G}>\mathrm{T}$ & p.Met1lle & 2 & missense variant & 1 & $0.70 \%$ \\
\hline c. $7 \mathrm{~A}>\mathrm{T}$ & p.Arg3Ter & 2 & missense variant & 1 & $0.70 \%$ \\
\hline c. $91 \mathrm{C}>\mathrm{T}$ & p.Arg31Ter & 2 & nonsense variant & 1 & $0.70 \%$ \\
\hline c. $103 \mathrm{C}>\mathrm{T}$ & p.GIn35Ter & 2 & nonsense variant & 2 & $1.40 \%$ \\
\hline c.144_145insA & p.Gln50Alafs $* 34$ & 2 & frameshift variant & 1 & $0.70 \%$ \\
\hline c. $323 \mathrm{G}>\mathrm{A}$ & p.Arg108His & 2 & missense variant & 9 & $6.29 \%$ \\
\hline$c .422 C>A$ & p.Ala141Glu & 3 & missense variant & 1 & $0.70 \%$ \\
\hline c. $424 \mathrm{~A}>\mathrm{G}$ & p.Thr142Ala & 3 & missense variant & 1 & $0.70 \%$ \\
\hline c. $425 \mathrm{C}>\mathrm{T}$ & p.Thr142lle & 3 & missense variant & 1 & $0.70 \%$ \\
\hline c. $433 \mathrm{G}>\mathrm{A}$ & p.Gly145Ser & 3 & missense variant & 1 & $0.70 \%$ \\
\hline c.445_446insA & p.Asp149Glufs*8 & 3 & frameshift variant & 1 & $0.70 \%$ \\
\hline c. $454 \mathrm{C}>\mathrm{T}$ & p.Arg152Ter & 3 & nonsense variant & 1 & $0.70 \%$ \\
\hline c. $467 \mathrm{~A}>\mathrm{T}$ & p.Asp156Val & 3 & missense variant & 1 & $0.70 \%$ \\
\hline c. $494 \mathrm{~A}>\mathrm{G}$ & p.Asp165Gly & 3 & missense variant & 1 & $0.70 \%$ \\
\hline c.567dupT & p.Gly190Trpfs*21 & 3 & frameshift variant & 1 & $0.70 \%$ \\
\hline c. $567 \mathrm{~T}>\mathrm{A}$ & p.Asn189Lys & 3 & missense variant & 1 & $0.70 \%$ \\
\hline c. $613 \mathrm{G}>\mathrm{A}$ & p.Glu205Lys & 3 & missense variant & 3 & $2.10 \%$ \\
\hline c. $616 \mathrm{C}>\mathrm{T}$ & p.GIn206Ter & 3 & nonsense variant & 1 & $0.70 \%$ \\
\hline c.626dupC & p.Lys210Ter & 3 & nonsense variant & 3 & $2.10 \%$ \\
\hline c. $652 \mathrm{C}>\mathrm{G}$ & p.GIn218Glu & 3 & missense variant & 1 & $0.70 \%$ \\
\hline c. $682 \mathrm{C}>\mathrm{T}$ & p.Arg228Ter & 3 & nonsense variant & 2 & $1.40 \%$ \\
\hline c.729_730insTT & p.Asp244Leufs*39 & 3 & frameshift variant & 24 & $16.78 \%$ \\
\hline c.755dupA & p.His252Glnfs*6 & 4 & frameshift variant & 6 & $4.20 \%$ \\
\hline c.791_797delACCATAT & p.Tyr264Cysfs*16 & 4 & frameshift variant & 1 & $0.70 \%$ \\
\hline $\mathrm{c.893T}>\mathrm{A}$ & p.lle298Asn & 4 & missense variant & 1 & $0.70 \%$ \\
\hline c. $914 \mathrm{~T}>\mathrm{C}$ & p.Leu305Ser & 5 & missense variant & 10 & $6.99 \%$ \\
\hline c.920_923delTCTT & p.Phe307Serfs*6 & 5 & frameshift variant & 1 & $0.70 \%$ \\
\hline c. $925 \mathrm{~T}>\mathrm{G}$ & p.Trp309Gly & 5 & missense variant & 1 & $0.70 \%$ \\
\hline c. $970 \mathrm{G}>\mathrm{A}$ & p.Ala324Thr & 5 & missense variant & 2 & $1.40 \%$ \\
\hline c. $1009 \mathrm{~T}>\mathrm{C}$ & p.Phe337Leu & 5 & missense variant & 1 & $0.70 \%$ \\
\hline c.1102delG & p.Val368Serfs*5 & 6 & frameshift variant & 1 & $0.70 \%$ \\
\hline c. $1105 \mathrm{C}>\mathrm{T}$ & p.Arg369Cys & 6 & missense variant & 3 & $2.10 \%$ \\
\hline c. $1106 \mathrm{G}>\mathrm{A}$ & p.Arg369His & 6 & missense variant & 11 & $7.69 \%$ \\
\hline
\end{tabular}




\begin{tabular}{|c|c|c|c|c|c|}
\hline cDNA change & amino acid change & exon & effect & frequency & percentage \\
\hline c.1107dupT & p.Thr370Tyrfs*22 & 6 & frameshift variant & 3 & $2.10 \%$ \\
\hline c. $1141 \mathrm{G}>\mathrm{A}$ & p.Gly381Arg & 6 & missense variant & 1 & $0.70 \%$ \\
\hline c. $1159 A>C$ & p.Thr387Pro & 6 & missense variant & 1 & $0.70 \%$ \\
\hline c. $1207 C>T$ & p.Arg403Ter & 6 & nonsense variant & 1 & $0.70 \%$ \\
\hline c.1233_1235delCAT & p.lle410del & 6 & deletion variant & 2 & $1.40 \%$ \\
\hline c. $1280 \mathrm{G}>\mathrm{A}$ & p.Gly427Asp & 6 & missense variant & 3 & $2.10 \%$ \\
\hline c. $1330 \mathrm{~A}>\mathrm{T}$ & p.Lys444Ter & 6 & nonsense variant & 1 & $0.70 \%$ \\
\hline c. $1420 \mathrm{C}>\mathrm{T}$ & p.Arg474Ter & 7 & nonsense variant & 2 & $1.40 \%$ \\
\hline c. $1531 \mathrm{C}>\mathrm{T}$ & p.Arg511Ter & 8 & nonsense variant & 1 & $0.70 \%$ \\
\hline$c .1560+2 T>C$ & & intron 8 & splicing variant & 1 & $0.70 \%$ \\
\hline c.1581_1582insA & p.Ala528Serfs*4 & 9 & frameshift variant & 1 & $0.70 \%$ \\
\hline c.1630_1631delGGinsTA & p.Gly544Ter & 9 & nonsense variant & 2 & $1.40 \%$ \\
\hline c.1645delC & p.Leu549Trpfs*21 & 9 & frameshift variant & 1 & $0.70 \%$ \\
\hline c. $1663 \mathrm{G}>\mathrm{A}$ & p.Ala555Thr & 9 & missense variant & 3 & $2.10 \%$ \\
\hline c. $1673 C>T$ & p.Ala558Val & 9 & missense variant & 1 & $0.70 \%$ \\
\hline c. $1675 A>G$ & p.Arg559Gly & 9 & missense variant & 1 & $0.70 \%$ \\
\hline c. $1677-1 \mathrm{G}>\mathrm{A}$ & & intron 9 & splicing variant & 4 & $2.80 \%$ \\
\hline c. $1678 \mathrm{~T}>\mathrm{C}$ & p.Cys560Arg & 10 & missense variant & 1 & $0.70 \%$ \\
\hline c. $1679 \mathrm{G}>\mathrm{A}$ & p.Cys560Tyr & 10 & missense variant & 1 & $0.70 \%$ \\
\hline c. $1741 \mathrm{C}>\mathrm{T}$ & p.Arg581Ter & 10 & nonsense variant & 2 & $1.40 \%$ \\
\hline c. $1777 \mathrm{G}>\mathrm{T}$ & p.Glu593Ter & 10 & nonsense variant & 1 & $0.70 \%$ \\
\hline c.1787delA & p.Glu596Glyfs*2 & 10 & frameshift variant & 1 & $0.70 \%$ \\
\hline c. $1880 A>G$ & p.His627Arg & 11 & missense variant & 2 & $1.40 \%$ \\
\hline c. $2080 \mathrm{C}>\mathrm{T}$ & p.Arg694Trp & 12 & missense variant & 2 & $1.40 \%$ \\
\hline c. $2179 \mathrm{C}>\mathrm{T}$ & p.Arg727Ter & 13 & nonsense variant & 4 & $2.80 \%$ \\
\hline Exon 13 Deletion & & 13 & deletion variant & 2 & $1.40 \%$ \\
\hline
\end{tabular}

Table 4

Other genes variants for 2 pedigrees

\begin{tabular}{|llllll|}
\hline Gene & cDNA change & amino acid change & exon & effect & frequency \\
Name & & & & \\
\hline LMBRD1 & c.981-2A $>$ TA & splicing region & splicing variant & 2 \\
SUCLG1 & c.826-2A $>$ G & splicing region & splicing variant & 2 \\
\hline
\end{tabular}

We summarize all the different types of variants occurred in our research, like missense variants, nonsense variants, splicing variants, small insertions, small deletions and large deletion (MUT exon 13 deletion). The spectrum of these distinct variants described in our study was distributed throughout the coding exons and splicing regions of the MMACHC and MUT genes. Many 
variants were occurred in exon 4 of $M M A C H C$ gene, and exon 3 of $M U T$ gene, respectively. And the frequency of these variants located in other exons were less.

\section{Prenatal Diagnosis And Follow-up}

Prenatal diagnosis was conducted by chorionic villus sampling of pregnant women, when variants were confirmed in the proband and proband's parents. Mathers' contamination was excluded by using a PowerPlex 16 HS System kit (Promega, Madison, WI, USA).

A total of 130 high-risk pregnancies in 130 families among all these pedigrees with clear MMACHC or MUT variants information choose to receive prenatal diagnosis. Among these 130 pedigrees, 22 foetuses were normal, 69 foetuses were carriers of heterozygous variants, and the remaining 39 foetuses harboured compound heterozygous variants or homozygous variants (Table 5and Table 6). Couples whose foetuses were normal or carriers choose to continue the pregnancy, however, for couples whose foetuses harboured homozygous variants or compound heterozygous variants they choose to terminate the pregnancy. The subsequent gene analysis of the foetuses' umbilical cord blood was in line with the prenatal diagnosis. Moreover, there are no abnormal findings in newborns screening by GC-MS. 
Table 5

The genotypes of $89 \mathrm{cblC}$ defect pedigrees who have performed the prenatal genetic diagnosis

\begin{tabular}{|c|c|c|c|c|}
\hline No.MMACHC & proband & $\begin{array}{l}\text { mutation } \\
\text { maternal }\end{array}$ & mutation paternal & fetus genotype \\
\hline 1 & c. $609 \mathrm{G} \square \mathrm{A} / \mathrm{c} .609 \mathrm{G} \square \mathrm{A}$ & c. $609 \mathrm{G} \square \mathrm{A}$ & c. $609 \mathrm{G} \square \mathrm{A}$ & $-/-$ \\
\hline 2 & c.609G $\square$ A/c.658_660delAAG & c. $609 \mathrm{G} \square \mathrm{A}$ & c.658_660delAAG & c.609GロA/c.658_660delAAG \\
\hline 3 & 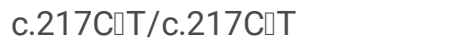 & c. $217 \mathrm{C} \square \mathrm{T}$ & c.217C $\square \mathrm{T}$ & c.217C口T/- \\
\hline 4 & c.609G $\square \mathrm{A} / \mathrm{c} .609 \mathrm{G} \square \mathrm{A}$ & c. $609 \mathrm{G} \square \mathrm{A}$ & c.609G $\square \mathrm{A}$ & 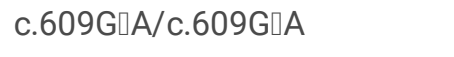 \\
\hline 5 & c.658_660delAAG/c.365A > T & c. $365 \mathrm{~A}>\mathrm{T}$ & c.658_660delAAG & c.658_660delAAG/- \\
\hline 6 & c.609GロA/c.445_446delTG & c.445_446delTG & c.609G $\square \mathrm{A}$ & c.609GロA/- \\
\hline 7 & $\begin{array}{l}\text { c.567_568insT/c.658_660del } \\
\text { AAG }\end{array}$ & c.567_568insT & c.658_660del AAG & $\begin{array}{l}\text { c.567_568insT/c.658_660del } \\
\text { AAG }\end{array}$ \\
\hline 8 & c.609G > A/c.567_568insT & c.567_568insT & c. $609 \mathrm{G} \square \mathrm{A}$ & c. $609 \mathrm{G}>\mathrm{A}$ \\
\hline 9 & c.609G > A/c.445_446delTG & c. $609 \mathrm{G}>\mathrm{A}$ & c.445_446delTG & c.609G > A/c.445_446delTG \\
\hline 10 & c. $609 \mathrm{G}>\mathrm{A} / \mathrm{c} .609 \mathrm{G}>\mathrm{A}$ & c. $609 \mathrm{G}>\mathrm{A}$ & c. $609 \mathrm{G}>\mathrm{A}$ & c. $609 \mathrm{G}>\mathrm{A} /-$ \\
\hline 11 & c. $80 A>G / c .609 G>A$ & c. $80 \mathrm{~A}>\mathrm{G}$ & c. $609 \mathrm{G}>\mathrm{A}$ & c. $80 \mathrm{~A}>\mathrm{G} /-$ \\
\hline 12 & c. $609 \mathrm{G}>\mathrm{A} / \mathrm{c} .683 \mathrm{C}>\mathrm{T}$ & c. $683 \mathrm{C}>\mathrm{T}$ & c. $609 \mathrm{G}>\mathrm{A}$ & $-/-$ \\
\hline 13 & $\begin{array}{l}\text { c.445_446delTG/c.658_660del } \\
\text { AAG }\end{array}$ & c.445_446delTG & c.658_660del AAG & c.445_446delTG/- \\
\hline 14 & c. $609 \mathrm{G}>\mathrm{A} / \mathrm{c} .599 \mathrm{G}>\mathrm{A}$ & c. $599 \mathrm{G}>\mathrm{A}$ & c. $609 \mathrm{G}>\mathrm{A}$ & c. $609 \mathrm{G}>\mathrm{A} /-$ \\
\hline 15 & c. $609 \mathrm{G}>\mathrm{A} / \mathrm{c} .617 \mathrm{G}>\mathrm{A}$ & c. $609 \mathrm{G}>\mathrm{A}$ & c. $617 \mathrm{G}>\mathrm{A}$ & c. $609 \mathrm{G}>\mathrm{A} / \mathrm{c} .617 \mathrm{G}>\mathrm{A}$ \\
\hline 16 & c. $80 \mathrm{~A}>\mathrm{G} / \mathrm{c} .482 \mathrm{G}>\mathrm{A}$ & c. $80 \mathrm{~A}>\mathrm{G}$ & c. $482 \mathrm{G}>\mathrm{A}$ & $\mathrm{c} .80 \mathrm{~A}>\mathrm{G} /-$ \\
\hline 17 & c.217C > T/c.658_660delAAG & c. $217 \mathrm{C}>\mathrm{T}$ & c.658_660delAAG & c.217C > T/c.658_660delAAG \\
\hline 18 & c. $609 \mathrm{G}>\mathrm{A} / \mathrm{c} .609 \mathrm{G}>\mathrm{A}$ & c. $609 \mathrm{G}>\mathrm{A}$ & c. $609 \mathrm{G}>\mathrm{A}$ & c. $609 \mathrm{G}>\mathrm{A} / \mathrm{c} .609 \mathrm{G}>\mathrm{A}$ \\
\hline 19 & c.463_465delGGG/c.609G > A & c.463_465delGGG & c. $609 \mathrm{G}>\mathrm{A}$ & $-/-$ \\
\hline 20 & c. $609 \mathrm{G}>\mathrm{A} / \mathrm{c} .467 \mathrm{G}>\mathrm{A}$ & c. $609 \mathrm{G}>\mathrm{A}$ & c. $467 \mathrm{G}>\mathrm{A}$ & c. $609 \mathrm{G}>\mathrm{A} / \mathrm{c} .467 \mathrm{G}>\mathrm{A}$ \\
\hline 21 & c.626dupT/c.609G >A & c.626dupT & c. $609 \mathrm{G}>\mathrm{A}$ & c.626dupT/- \\
\hline 22 & c.658_660delAAG/c.609G > A & $\begin{array}{l}\text { c. } 658 \_660 \\
\text { delAAG }\end{array}$ & c. $609 \mathrm{G}>\mathrm{A}$ & c.658_660delAAG/c.609G >A \\
\hline 23 & c.626dupC/c. $1106 \mathrm{G}>\mathrm{A}$ & c.626dupC & c. $1106 \mathrm{G}>\mathrm{A}$ & c.626dupC/c. $1106 \mathrm{G}>\mathrm{A}$ \\
\hline 24 & c.445_446delTG/c.609G >A & c.445_446delTG & c. $609 \mathrm{G}>\mathrm{A}$ & c.445_446delTG/c.609G > A \\
\hline 25 & c. $394 \mathrm{C}>\mathrm{T} / \mathrm{c} .609 \mathrm{G}>\mathrm{A}$ & c. $394 \mathrm{C}>\mathrm{T}$ & c. $609 \mathrm{G}>\mathrm{A}$ & c. $394 \mathrm{C}>\mathrm{T} /-$ \\
\hline 26 & c. $609 \mathrm{G}>\mathrm{A} / \mathrm{c} .637 \mathrm{G}>\mathrm{T}$ & c. $637 \mathrm{G}>\mathrm{T}$ & c. $609 \mathrm{G}>\mathrm{A}$ & c. $609 \mathrm{G}>\mathrm{A} /-$ \\
\hline 27 & $\begin{array}{l}\text { c.658_660delAAG } \\
\text { /c.658_660delAAG }\end{array}$ & c.658_660delAAG & c.658_660delAAG & $\begin{array}{l}\text { c.658_660delAAG } \\
\text { /c.658_660delAAG }\end{array}$ \\
\hline 28 & c. $609 \mathrm{G}>\mathrm{A} / \mathrm{c} .609 \mathrm{G}>\mathrm{A}$ & c. $609 \mathrm{G}>\mathrm{A}$ & c. $609 \mathrm{G}>\mathrm{A}$ & c. $609 \mathrm{G}>\mathrm{A} /-$ \\
\hline 29 & c. $481 \mathrm{C}>\mathrm{T} / \mathrm{c} .609 \mathrm{G}>\mathrm{A}$ & c. $.481 \mathrm{C}>\mathrm{T}$ & c. $609 \mathrm{G}>\mathrm{A}$ & $-/-$ \\
\hline 30 & c. $609 \mathrm{G}>\mathrm{A} / \mathrm{c} .609 \mathrm{G}>\mathrm{A}$ & c. $609 \mathrm{G}>\mathrm{A}$ & c. $609 \mathrm{G}>\mathrm{A}$ & c. $609 \mathrm{G}>\mathrm{A} /-$ \\
\hline 31 & c.658_660delAAG/c.567dupT & c.658_660delAAG & c.567dupT & c.658_660delAAG/- \\
\hline
\end{tabular}




\begin{tabular}{|c|c|c|c|c|}
\hline No.MMACHC & proband & $\begin{array}{l}\text { mutation } \\
\text { maternal }\end{array}$ & mutation paternal & fetus genotype \\
\hline 32 & c. $394 \mathrm{C}>\mathrm{T} / \mathrm{c} .615 \mathrm{C}>\mathrm{A}$ & c. $394 \mathrm{C}>\mathrm{T}$ & c. $615 \mathrm{C}>\mathrm{A}$ & c. $394 \mathrm{C}>\mathrm{T} / \mathrm{c} .615 \mathrm{C}>\mathrm{A}$ \\
\hline 33 & c.567dupT/c.445_446insA & c.445_446insA & c.567dupT & c.567dupT/- \\
\hline 34 & c. $609 \mathrm{G}>\mathrm{A} / \mathrm{c} .217 \mathrm{C}>\mathrm{T}$ & c. $217 \mathrm{C}>\mathrm{T}$ & c. $609 \mathrm{G}>\mathrm{A}$ & c. $609 \mathrm{G}>\mathrm{A} /-$ \\
\hline 35 & c.567dupT/c.609G >A & c.567dupT & c. $609 \mathrm{G}>\mathrm{A}$ & c.567dupT/c.609G >A \\
\hline 36 & c.609G > A/c.656_658delAAG & c. $609 \mathrm{G}>\mathrm{A}$ & c.656_658delAAG & c. $609 \mathrm{G}>\mathrm{A} /-$ \\
\hline 37 & c. $467 \mathrm{G}>\mathrm{A} / \mathrm{c} .609 \mathrm{G}>\mathrm{A}$ & c. $467 \mathrm{G}>\mathrm{A}$ & c. $609 \mathrm{G}>\mathrm{A}$ & c. $467 \mathrm{G}>\mathrm{A} /-$ \\
\hline 38 & c. $609 \mathrm{G}>\mathrm{A} / \mathrm{c} .609 \mathrm{G}>\mathrm{A}$ & c. $609 \mathrm{G}>\mathrm{A}$ & c. $609 \mathrm{G}>\mathrm{A}$ & c. $609 \mathrm{G}>\mathrm{A} /-$ \\
\hline 39 & c. $609 \mathrm{G}>\mathrm{A} / \mathrm{c} .481 \mathrm{C}>\mathrm{T}$ & c. $481 \mathrm{C}>\mathrm{T}$ & c. $609 \mathrm{G}>\mathrm{A}$ & c. $609 \mathrm{G}>\mathrm{A} /-$ \\
\hline 40 & c.394C > T/c.567dupT & c.567dupT & c. $394 \mathrm{C}>\mathrm{T}$ & c.394C > T/- \\
\hline 41 & c. $609 \mathrm{G}>\mathrm{A} / \mathrm{c} .609 \mathrm{G}>\mathrm{A}$ & c. $609 \mathrm{G}>\mathrm{A}$ & c. $609 \mathrm{G}>\mathrm{A}$ & c. $609 \mathrm{G}>\mathrm{A} /-$ \\
\hline 42 & c.609G > A/c.440_441delGT & c. $609 \mathrm{G}>\mathrm{A}$ & c.440_441delGT & c. $609 \mathrm{G}>\mathrm{A} /-$ \\
\hline 43 & c.609G > A/c.331C > T & c. $331 \mathrm{C}>\mathrm{T}$ & c. $609 \mathrm{G}>\mathrm{A}$ & c. $609 \mathrm{G}>\mathrm{A} /-$ \\
\hline 44 & c.658_660 delAAG/c.609G >A & $\begin{array}{l}\text { c. } 658 \_660 \\
\text { delAAG }\end{array}$ & c. $609 \mathrm{G}>\mathrm{A}$ & c.658_660 delAAG/c.609G > A \\
\hline 45 & c.80A > G/c.658_660delAAG & c. $80 A>G$ & c.658_660delAAG & c.80A > G/c.658_660delAAG \\
\hline 46 & c.609G > A/c.567dupT & c.567dupT & c. $609 \mathrm{G}>\mathrm{A}$ & c. $609 \mathrm{G}>\mathrm{A} /-$ \\
\hline 47 & $\mathrm{c} .80 \mathrm{~A}>\mathrm{G} / \mathrm{c} .80 \mathrm{~A}>\mathrm{G}$ & c. $80 A>G$ & c. $80 \mathrm{~A}>\mathrm{G}$ & c. $80 \mathrm{~A}>\mathrm{G} / \mathrm{c} .80 \mathrm{~A}>\mathrm{G}$ \\
\hline 48 & c.567dupT/c.658_660delAAG & c.567dupT & c.658_660delAAG & $-/-$ \\
\hline 49 & c. $666 \mathrm{C}>\mathrm{A} / \mathrm{c} .80 \mathrm{~A}>\mathrm{G}$ & c. $666 \mathrm{C}>\mathrm{A}$ & c. $80 A>G$ & c. $666 \mathrm{C}>\mathrm{A} /-$ \\
\hline 50 & c.609G > A/c.658_660delAAG & c.658_660delAAG & c. $609 \mathrm{G}>\mathrm{A}$ & c. $609 \mathrm{G}>\mathrm{A} /-$ \\
\hline 51 & c.609G > A/c.658_660delAAG & c. $609 \mathrm{G}>\mathrm{A}$ & c.658_660delAAG & $-/-$ \\
\hline 52 & c.658_660delAAG/c.609G >A & c.658_660delAAG & c. $609 \mathrm{G}>\mathrm{A}$ & $-/-$ \\
\hline 53 & c. $80 A>G / c .609 G>A$ & c. $80 A>G$ & c. $609 \mathrm{G}>\mathrm{A}$ & $-/-$ \\
\hline 54 & c.658_660delAAG/c.609 > A & c. $609>\mathrm{A}$ & c.658_660delAAG & c.658_660delAAG/- \\
\hline 55 & c. $609 \mathrm{G}>\mathrm{A} / \mathrm{c} .482 \mathrm{G}>\mathrm{A}$ & c. $609 \mathrm{G}>\mathrm{A}$ & c. $482 \mathrm{G}>\mathrm{A}$ & c. $609 \mathrm{G}>\mathrm{A} / \mathrm{c} .482 \mathrm{G}>\mathrm{A}$ \\
\hline 56 & c. $609 \mathrm{G}>\mathrm{A} / \mathrm{c} .609 \mathrm{G}>\mathrm{A}$ & c. $609 \mathrm{G}>\mathrm{A}$ & c. $609 \mathrm{G}>\mathrm{A}$ & c. $609 \mathrm{G}>\mathrm{A} / \mathrm{c} .609 \mathrm{G}>\mathrm{A}$ \\
\hline 57 & c.658_660delAAG/c.609G >A & c. $609 \mathrm{G}>\mathrm{A}$ & c.658_660delAAG & c.658_660delAAG/- \\
\hline 58 & c.609G > A/c.445_446insA & c. $609 \mathrm{G}>\mathrm{A}$ & c.445_446insA & c.609G > A/c.445_446insA \\
\hline 59 & c. $609 \mathrm{G}>\mathrm{A} / \mathrm{c} .609 \mathrm{G}>\mathrm{A}$ & c. $609 \mathrm{G}>\mathrm{A}$ & c. $609 \mathrm{G}>\mathrm{A}$ & c. $609 \mathrm{G}>\mathrm{A} /-$ \\
\hline 60 & c.80A > G/c.566_567insT & c. $80 A>G$ & c.566_567insT & c.80A > G/c.566_567insT \\
\hline 61 & c. $609 \mathrm{G}>\mathrm{A} / \mathrm{c} .609 \mathrm{G}>\mathrm{A}$ & c. $609 \mathrm{G}>\mathrm{A}$ & c. $609 \mathrm{G}>\mathrm{A}$ & c. $609 \mathrm{G}>\mathrm{A} /-$ \\
\hline 62 & c. $609 \mathrm{G}>\mathrm{A} / \mathrm{c} .80 \mathrm{~A}>\mathrm{G}$ & c. $80 A>G$ & c. $609 \mathrm{G}>\mathrm{A}$ & c. $609 \mathrm{G}>\mathrm{A} /-$ \\
\hline 63 & c. $217 \mathrm{C}>\mathrm{T} / \mathrm{c} .609 \mathrm{G}>\mathrm{A}$ & c. $217 \mathrm{C}>\mathrm{T}$ & c. $609 \mathrm{G}>\mathrm{A}$ & $-/$ \\
\hline 64 & c. $609 \mathrm{G}>\mathrm{A} / \mathrm{c} .217 \mathrm{C}>\mathrm{T}$ & c. $609 \mathrm{G}>\mathrm{A}$ & c. $217 \mathrm{C}>\mathrm{T}$ & $-/-$ \\
\hline 65 & c.609G > A/c.658_660delAAG & c.658_660delAAG & c. $609 \mathrm{G}>\mathrm{A}$ & c. $609 \mathrm{G}>\mathrm{A} /-$ \\
\hline
\end{tabular}




\begin{tabular}{|c|c|c|c|c|}
\hline No.MMACHC & proband & $\begin{array}{l}\text { mutation } \\
\text { maternal }\end{array}$ & mutation paternal & fetus genotype \\
\hline 66 & c. $609 \mathrm{G}>\mathrm{A} / \mathrm{c} .609 \mathrm{G}>\mathrm{A}$ & c. $609 \mathrm{G}>\mathrm{A}$ & c. $609 \mathrm{G}>\mathrm{A}$ & c. $609 \mathrm{G}>\mathrm{A} /-$ \\
\hline 67 & c.609G > A/c.658_660delAAG & c.658_660delAAG & c. $609 \mathrm{G}>\mathrm{A}$ & c. $609 \mathrm{G}>\mathrm{A} /-$ \\
\hline 68 & c. $80 A>G / c .609 G>A$ & c. $80 A>G$ & c. $609 \mathrm{G}>\mathrm{A}$ & c. $80 \mathrm{~A}>\mathrm{G} / \mathrm{c} .609 \mathrm{G}>\mathrm{A}$ \\
\hline 69 & c.658_660delAAG/c.609G > A & c. $609 \mathrm{G}>\mathrm{A}$ & c.658_660delAAG & c.658_660delAAG/- \\
\hline 70 & c. $609 \mathrm{G}>\mathrm{A} / \mathrm{c} .567 \mathrm{dup} T$ & c. $609 \mathrm{G}>\mathrm{A}$ & c.567dupT & $-/$ \\
\hline 71 & c.609G > A/c.658_660delAAG & c. $609 \mathrm{G}>\mathrm{A}$ & c.658_660delAAG & c.609G > A/c.658_660delAAG \\
\hline 72 & c.609G > A/c.658_660delAAG & c. $609 \mathrm{G}>\mathrm{A}$ & c.658_660delAAG & $-/-$ \\
\hline 73 & c.567dupT/c.626dupT & c.567dupT & c.626dupT & c.567dupT/- \\
\hline 74 & c.658_660delAAG/c.609G > A & c.658_660delAAG & c. $609 \mathrm{G}>\mathrm{A}$ & c.658_660delAAG/- \\
\hline 75 & c. $609 \mathrm{G}>\mathrm{A} / \mathrm{c} .567 \mathrm{dup} T$ & c.567dupT & c. $609 \mathrm{G}>\mathrm{A}$ & c. $609 \mathrm{G}>\mathrm{A} /-$ \\
\hline 76 & c. $615 \mathrm{C}>\mathrm{A} / \mathrm{c} .609 \mathrm{G}>\mathrm{A}$ & c. $615 \mathrm{C}>\mathrm{A}$ & c. $609 \mathrm{G}>\mathrm{A}$ & c. $615 \mathrm{C}>\mathrm{A} / \mathrm{c} .609 \mathrm{G}>\mathrm{A}$ \\
\hline 77 & c. $89 \mathrm{G}>\mathrm{A} / \mathrm{c} .567 \mathrm{dup} T$ & c. $89 \mathrm{G}>\mathrm{A}$ & c.567dupT & c. $89 \mathrm{G}>\mathrm{A} /-$ \\
\hline 78 & c.567dupT/c.658_660delAAG & c.567dupT & c.658_660delAAG & c.567dupT/- \\
\hline 79 & c. $609 \mathrm{G}>\mathrm{A} / \mathrm{c} .315 \mathrm{C}>\mathrm{G}$ & c. $609 \mathrm{G}>\mathrm{A}$ & c. $315 C>G$ & c. $609 \mathrm{G}>\mathrm{A} /-$ \\
\hline 80 & c.567dupT/c.482G > A & c. $482 \mathrm{G}>\mathrm{A}$ & c.567dupT & c.567dupT/- \\
\hline 81 & c. $315 \mathrm{C}>\mathrm{G} / \mathrm{c} .609 \mathrm{G}>\mathrm{A}$ & c. $315 \mathrm{C}>\mathrm{G}$ & c. $609 \mathrm{G}>\mathrm{A}$ & c. $315 \mathrm{C}>\mathrm{G} /-$ \\
\hline 82 & c. $315 \mathrm{C}>\mathrm{G} / \mathrm{c} .609 \mathrm{G}>\mathrm{A}$ & c. $315 C>G$ & c. $609 \mathrm{G}>\mathrm{A}$ & $-/-$ \\
\hline 83 & c. $609 \mathrm{G}>\mathrm{A} / \mathrm{c} .394 \mathrm{C}>\mathrm{T}$ & c. $609 \mathrm{G}>\mathrm{A}$ & c. $394 \mathrm{C}>\mathrm{T}$ & c. $609 \mathrm{G}>\mathrm{A} /-$ \\
\hline 84 & c. $440 \mathrm{G}>\mathrm{A} / \mathrm{c} .609 \mathrm{G}>\mathrm{A}$ & c. $609 \mathrm{G}>\mathrm{A}$ & c. $440 \mathrm{G}>\mathrm{A}$ & c. $440 \mathrm{G}>\mathrm{A} /-$ \\
\hline 85 & c.609G > A/c.217C > T & c. $217 \mathrm{C}>\mathrm{T}$ & c. $609 \mathrm{G}>\mathrm{A}$ & c. $609 \mathrm{G}>\mathrm{A} /-$ \\
\hline 86 & c. $609 \mathrm{G}>\mathrm{A} / \mathrm{c} .80 \mathrm{~A}>\mathrm{G}$ & $\mathrm{c} .80 \mathrm{~A}>\mathrm{G}$ & c. $609 \mathrm{G}>\mathrm{A}$ & c. $609 \mathrm{G}>\mathrm{A} /-$ \\
\hline 87 & c. $609 \mathrm{G}>\mathrm{A} / \mathrm{c} .609 \mathrm{G}>\mathrm{A}$ & c. $609 \mathrm{G}>\mathrm{A}$ & c. $609 \mathrm{G}>\mathrm{A}$ & c. $609 \mathrm{G}>\mathrm{A} /-$ \\
\hline 88 & c.415_416delinsTA/c.567dupT & c.567dupT & c.415_416delinsTA & c.415_416delinsTA/- \\
\hline 89 & c.609G > A/c.658_660delAAG & c. $609 \mathrm{G}>\mathrm{A}$ & c.658_660delAAG & c.609G > A/c.658_660delAAG \\
\hline
\end{tabular}


Table 6

The genotypes of 41 MUT type pedigrees who have performed the prenatal genetic diagnosis

\begin{tabular}{|c|c|c|c|c|}
\hline No.MUT & proband & mutation maternal & mutation paternal & fetus genotype \\
\hline 1 & c. $1280 \mathrm{G}>\mathrm{A} / \mathrm{c} .616 \mathrm{C}>\mathrm{T}$ & c. $616 \mathrm{C}>\mathrm{T}$ & c. $1280 G>A$ & c. $1280 \mathrm{G}>\mathrm{A} /-$ \\
\hline 2 & c. $1675 \mathrm{~A}>$ G/c.729_730 ins TT & c. $1675 A>G$ & c.729_730 ins TT & $-/$ \\
\hline 3 & c. $323 \mathrm{G}>\mathrm{A} / \mathrm{c} .323 \mathrm{G}>\mathrm{A}$ & c. $323 G>A$ & c. $323 \mathrm{G}>\mathrm{A}$ & $-/-$ \\
\hline 4 & c.914T >C/c.567T >A & c. $914 \mathrm{~T}>\mathrm{C}$ & c. $567 \mathrm{~T}>\mathrm{A}$ & c. $914 \mathrm{~T}>\mathrm{C} /-$ \\
\hline \multirow[t]{2}{*}{5} & c.729_730insTT/ & c.729_730insTT & c.729_730insTT & c.729_730insTT/ \\
\hline & c.729_730insTT & & & c.729_730insTT \\
\hline 6 & c.626_627insC/c.1679G >A & c. $1679 \mathrm{G}>\mathrm{A}$ & c.626_627insC & c.626_627insC/- \\
\hline 7 & c.914T > C/c.757_758insA & c.757_758insA & c. $914 \mathrm{~T}>\mathrm{C}$ & c. $914 \mathrm{~T}>\mathrm{C} /-$ \\
\hline 8 & c. $925 \mathrm{~T}>\mathrm{G} / \mathrm{c} .1106 \mathrm{G}>\mathrm{A}$ & c. $925 \mathrm{~T}>\mathrm{G}$ & c. $1106 \mathrm{G}>\mathrm{A}$ & c. $925 \mathrm{~T}>\mathrm{G} / \mathrm{c} .1106 \mathrm{G}>\mathrm{A}$ \\
\hline 9 & c. $1106 \mathrm{G}>\mathrm{A} / \mathrm{c} .91 \mathrm{C}>\mathrm{T}$ & c. $1106 \mathrm{G}>\mathrm{A}$ & c. $91 \mathrm{C}>\mathrm{T}$ & c. $1106 \mathrm{G}>\mathrm{A} /-$ \\
\hline \multirow[t]{2}{*}{10} & c.729_730insTT/ & c.729_730insTT & c.756_757insA & c.729_730insTT/- \\
\hline & c.756_757insA & & & \\
\hline 11 & c.729_730insTT/c.1280G > A & c.729_730insTT & c. $1280 G>A$ & $-/-$ \\
\hline 12 & c. $1106 \mathrm{G}>\mathrm{A} / \mathrm{c} .914 \mathrm{~T}>\mathrm{C}$ & c. $914 \mathrm{~T}>\mathrm{C}$ & c. $1106 \mathrm{G}>\mathrm{A}$ & c. $1106 \mathrm{G}>\mathrm{A} /-$ \\
\hline 13 & c. $422 \mathrm{C}>\mathrm{A} / \mathrm{c} .424 \mathrm{~A}>\mathrm{G}$ & c. $422 \mathrm{C}>\mathrm{A}$ & c. $424 \mathrm{~A}>\mathrm{G}$ & $-/-$ \\
\hline 14 & c. $323 \mathrm{G}>\mathrm{A} / \mathrm{c} .1420 \mathrm{C}>\mathrm{T}$ & c. $1420 \mathrm{C}>\mathrm{T}$ & c. $323 \mathrm{G}>\mathrm{A}$ & c. $323 \mathrm{G}>\mathrm{A} /-$ \\
\hline 15 & c. $1560+2 T>C / c .1105 C>T$ & c. $1560+2 \mathrm{~T}>\mathrm{C}$ & c. $1105 \mathrm{C}>\mathrm{T}$ & $-/$ \\
\hline 16 & c. $1106 \mathrm{G}>\mathrm{A} / \mathrm{c} .1102 \mathrm{del} \mathrm{G}$ & c. $1106 \mathrm{G}>\mathrm{A}$ & c.1102delG & $-/$ \\
\hline 17 & c. $613 \mathrm{G}>\mathrm{A} / \mathrm{c} .1106 \mathrm{G}>\mathrm{A}$ & c. $1106 \mathrm{G}>\mathrm{A}$ & c. $613 \mathrm{G}>\mathrm{A}$ & c. $613 \mathrm{G}>\mathrm{A} /-$ \\
\hline 18 & c. $323 \mathrm{G}>\mathrm{A} / \mathrm{c} .1531 \mathrm{C}>\mathrm{T}$ & c. $323 \mathrm{G}>\mathrm{A}$ & c. $1531 \mathrm{C}>\mathrm{T}$ & c. $323 \mathrm{G}>\mathrm{A} / \mathrm{c} .1531 \mathrm{C}>\mathrm{T}$ \\
\hline 19 & c. $1105 \mathrm{C}>\mathrm{T} / \mathrm{c} .652 \mathrm{C}>\mathrm{G}$ & c. $1105 \mathrm{C}>\mathrm{T}$ & c. $652 C>G$ & c. $1105 \mathrm{C}>\mathrm{T} / \mathrm{c} .652 \mathrm{C}>\mathrm{G}$ \\
\hline 20 & c. $626 \mathrm{dupC} / \mathrm{c} .1106 \mathrm{G}>\mathrm{A}$ & c.626dupC & c. $1106 \mathrm{G}>\mathrm{A}$ & c.626dupC/c.1106G >A \\
\hline \multirow[t]{2}{*}{21} & c.920_923delTCTT/ & c.920_923delTCTT & c.791_797delACCATAT & c.920_923delTCTT/ \\
\hline & c.791_797delACCATAT & & & c.791_797delACCATAT \\
\hline 22 & c.1107dupT/c.1107dupT & c.1107dupT & c.1107dupT & c.1107dupT/c.1107dupT \\
\hline 23 & c. $2080 \mathrm{C}>\mathrm{T} / \mathrm{E} 13$ deletion & c. $2080 \mathrm{C}>\mathrm{T}$ & MUT Exon 13 deletion & c. $2080 \mathrm{C}>\mathrm{T} /-$ \\
\hline 24 & c.567dupT/c.445_446insA & c.445_446insA & c.567dupT & c.567dupT/- \\
\hline \multirow[t]{2}{*}{25} & c. $1207 \mathrm{C}>\mathrm{T} /$ & c. $1207 \mathrm{C}>\mathrm{T}$ & c.1630_1631delGGinsTA & c. $1207 \mathrm{C}>\mathrm{T} /$ \\
\hline & c.1630_1631delGGinsTA & & & c.1630_1631delGGinsTA \\
\hline 26 & c. $103 \mathrm{C}>\mathrm{T} / \mathrm{c} .1106 \mathrm{G}>\mathrm{A}$ & c. $103 \mathrm{C}>\mathrm{T}$ & c. $1106 \mathrm{G}>\mathrm{A}$ & $-/-$ \\
\hline 27 & c.1741C > T /c.729_730insTT & c. $1741 \mathrm{C}>\mathrm{T}$ & c.729_730insTT & $-/-$ \\
\hline 28 & c.1106G > A/c.1787_1787delA & c. $1106 \mathrm{G}>\mathrm{A}$ & c.1787_1787delA & c. $1106 \mathrm{G}>\mathrm{A} /-$ \\
\hline 29 & c.144_145insA/c.755_756insA & c.144_145insA & c.755_756insA & c.144_145insA/c.755_756insA \\
\hline 30 & c. $1677-1 \mathrm{G}>\mathrm{A} / \mathrm{c} .1677-1 \mathrm{G}>\mathrm{A}$ & c. $1677-1 G>A$ & c. $1677-1 \mathrm{G}>\mathrm{A}$ & c. $1677-1 \mathrm{G}>\mathrm{A} /-$ \\
\hline
\end{tabular}




\begin{tabular}{|c|c|c|c|c|}
\hline No.MUT & proband & mutation maternal & mutation paternal & fetus genotype \\
\hline 31 & c.729_730insTT/c.1880A > G & c.729_730insTT & c. $1880 A>G$ & c.729_730insTT/c.1880A > G \\
\hline 32 & c.2179C > T/c.729_730insTT & c. $2179 \mathrm{C}>\mathrm{T}$ & c.729_730insTT & c.2179C > T/c.729_730insTT \\
\hline 33 & c. $2179 \mathrm{C}>\mathrm{T} / \mathrm{c} .425 \mathrm{C}>\mathrm{T}$ & c. $2179 \mathrm{C}>\mathrm{T}$ & c. $425 \mathrm{C}>\mathrm{T}$ & $-/-$ \\
\hline \multirow[t]{2}{*}{34} & c.1233_1235delCAT/ & c.1233_1235delCAT & c. $1105 \mathrm{C}>\mathrm{T}$ & c.1233_1235delCAT/- \\
\hline & c. $1105 \mathrm{C}>\mathrm{T}$ & & & \\
\hline 35 & c. $914 \mathrm{~T}>\mathrm{C} / \mathrm{c} .1741 \mathrm{C}>\mathrm{T}$ & c. $914 \mathrm{~T}>\mathrm{C}$ & c. $1741 \mathrm{C}>\mathrm{T}$ & c. $914 \mathrm{~T}>\mathrm{C} / \mathrm{c} .1741 \mathrm{C}>\mathrm{T}$ \\
\hline 36 & c. $-39-2 A>G / c .1678 T>C$ & c. $-39-2 A>G$ & c. $1678 \mathrm{~T}>\mathrm{C}$ & c. $-39-2 A>G / c .1678 T>C$ \\
\hline 37 & c. $914 \mathrm{~T}>\mathrm{C} / \mathrm{c} .970 \mathrm{G}>\mathrm{A}$ & c. $914 \mathrm{~T}>\mathrm{C}$ & c. $970 \mathrm{G}>\mathrm{A}$ & c.914T > C/- \\
\hline 38 & c. $454 \mathrm{C}>\mathrm{T} / \mathrm{c} .2080 \mathrm{C}>\mathrm{T}$ & c. $2080 \mathrm{C}>\mathrm{T}$ & c. $454 \mathrm{C}>\mathrm{T}$ & c. $454 \mathrm{C}>\mathrm{T} /-$ \\
\hline 39 & c. $433 \mathrm{G}>\mathrm{A} / \mathrm{c} .7 \mathrm{~A}>\mathrm{T}$ & c.7A $>\mathrm{T}$ & c. $433 \mathrm{G}>\mathrm{A}$ & c. $433 \mathrm{G}>\mathrm{A} /-$ \\
\hline 40 & c.467A > T/c.729_730insTT & c. $467 \mathrm{~A}>\mathrm{T}$ & c.729_730insTT & c.467A > T/c.729_730insTT \\
\hline 41 & c. $1159 \mathrm{~A}>\mathrm{C} / \mathrm{c} .914 \mathrm{~T}>\mathrm{C}$ & c. $1159 A>C$ & c. $914 \mathrm{~T}>\mathrm{C}$ & c. $1159 \mathrm{~A}>\mathrm{C} /-$ \\
\hline
\end{tabular}

\section{Discussion}

MMA, which refers to a cohort of organic aciduria caused by deficiency in methylmalonyl coenzyme A mutase (MCM) or intracellular cobalamin (cbl) metabolism, is the most common form of organic aciduria in the mainland of China. Recently, thanks to newborn screening by MS-MS or GC-MS, an increasing number of MMA patients could get early diagnosis and effective treatment. However, the long-term outcomes are not encouraging, along with severe neurological and renal complications. Therefore it is very important to prevent the recurrence in families with MMA child birth history. This study reports prenatal molecular genetic diagnosis for 130 pedigrees with clear gene variants as early as the first trimester by chorionic villus sampling, which is the best currently available prevention method.

Among the 244 pedigrees, 168 (68.9\%) of which were combined MMA, the remaining 76 (31.1\%) were isolated MMA, showing that MMA combined with hyperhomocystinuria is the major biochemical phenotype, and their proportion approaches what have been reported in other literatures [18,32]. Most of the patients who were clinically diagnosed other than identified via newborn screening present in the first year and typically in the neonatal period.

For the isolated MMA, severe metabolic crises were very common, which is rarely seen in cblC patiens. Neuropsychiatric damages like seizure and hypotonia are frequently found in these patients, which could also occur in cblC patients. other neural complications like feeding problems, delayed development and intellectual disability could be seen both in isolated and combined MMA. Renal insufficiency is frequently reported in mutase-deficient methylmalonic academia, and they are susceptible to metabolic strokes. While pulmonary arterial hypertensions and hemolytic uremic syndrome could occur in cblC patients. Less common features of combined MMA include eye problems and megaloblastic anemia, but rare in isolated patients.

One patient with mitochondrial DNA depletion sysdrome (MDS) caused by SUCLG1 gene variants had lower blood methylmalonic acid than others patients, but he got severe neurological damages. One pedigree with diseased proband was clinically diagnosed MMA with high methylmalonic acid in the urine, the parents both harboured the c.981-2A > TA heterozygous variant in $L M B R D 1$ gene(CbIF).

In a total of 244 families, only 20 children were discovered by newborn screening. The remaining were diagnosed based on the probands' clinical manifestations, and the results of MS-MS and (or) GC-MS. They all suffer much pain because of MMA and usually lost optimal treatment opportunities, until they were clearly diagnosed and got prompt treatment. However, the prognosis of those 20 patients detected by neonatal screening is very good, which indicated that neonatal screening is a key strategy of early diagnosis and effective treatment. Cobalamin and levocarnitine were all used to treat MMA. The therapy of isolated MMA relies on stopping protein intake, whereas this is contra-indicated in cblC patients, who have low methionine levels.

Page $14 / 20$ 
In our research, there are 244 probands who received sequencing analysis, and we found different pathogenic variants in 4 genes including MMACHC, MUT, LMBRD1 and SUCLG1, among which MMACHC and MUT gene variants are the most prevalent, verifying the complexity of genotypes in Chinese MMA patients. For 8 probands with negative gene variants and 6 probands with only one heterozygous gene variant, they were all detected by Next Generation Sequencing, which may be due to variations outside the scope of current techniques or caused by other genes which is outside the range of genes in our NGS inherited metabolic disorders panel..

Among the MMA patients with genetic diagnosis, 158 cases were MMACHC gene mutations, which confirmed that cblC type is the main type of MMA with homocysteinemia in China. Of the 76 isolated MMAs that received genetic analysis, 70 (92.1\%) were MUT gene variants, confirming that the mutase deficiency caused by MUT gene deficiency is the main type of simple MMA in China. At the same time, MMA caused by 1 cases of $\angle M B R D 1,1$ case of SUCLG1 gene defect were found, suggesting that cblF type MMA is a rare type in China, which are different from other countries. Other genes defect like SUCLG1 and SUCLA2, which could cause mitochondrial DNA depletion sysdrome (MDS) accompanied by the phenotype of methylmalonic acid in urine and blood, should also come into our consideration in clinical practice.

To date, it has been reported more than 90 variants in $M M A C H C$ gene and 250 variants in $M U T$ gene in the literature, respectively, including missense mutation, nonsense mutation, small insertions and deletions, splicing region mutation et al [33, 34]. In view of the high allelic heterogeneity in MMA, the spectrums of variants in MMACHC and MUT gene are different in various populations around the world, suggesting that mutation analysis of MMA patients is significant in China. Our research shows that c. $609 \mathrm{G}>\mathrm{A}$ (48.28\%), c.658_660delAAG (13.17\%) and c.567_568insT (7.84\%) are the most frequent variants for cblC defect patients. And for MUT type patients, c.729_730insTT (16.78\%), c.1106G > A (7.69\%), c.914T > C (6.99\%) and c.323G >A (6.29\%) are more frequent than other variants.

It has been illustrated that for cblC type patients, c.271dupA were the most common allele in different European countries from their study [35, 36]. Other literature had indicated that c.394C > T variant is the most frequent variants in Native American and Middle Eastern MMA patients [36, 37]. For East Asians, a great number of cblC defect patients were homozygous in c.609G >A variant [30]. Lots of Chinese study shows that C.609G > A was the most common mutation in Chinese cblC defect patients [38, 39].

Similarily, in our study, among 38 homozygous variants, 34 of them were homozygous for c.609G > A, which accounts for $48.28 \%$. Therefore, we can further confirm that the $.609 \mathrm{G}>\mathrm{A}$ variant is the most common mutation in Chinese population. It was followed by c.658_660delAAG and c.567_568insT variants, which accounted for $13.17 \%$ and $7.84 \%$, respectively, and these two variants are very common mutation in Chinese patients.

For MUT type patients, considering the wide spectrum of variants, their distribution is heterogeneous from exon 1 to exon 13 . Thus, mutation analysis is crucial in different populations. The c. $278 \mathrm{G}>\mathrm{A}$ and c.329A $>\mathrm{G}$ are the most frequent variants in Saudi Arabian patients based on a study conducted in 2014 [40]. The c.322C > T mutation present in 41\% of black patients [41] and 60\% of Hispanic patients [42]. The mutation c.731A > T was the first frequent mutation reported in Caucasian population [43], c.425G > T in Japanese patients [44], and c.1280G > A and c.1630_1631delGGinsTA are the frequent mutations in Asian patients [42]. A Chinese research in 2012 shows that c.729_730insTT and c.1280G > A were the most frequent variants in the mut-type MMA patients [45]. Another Chinese article revealed that c.323G > A, c.729_730insTT and c.1630_1631delGGinsTA were more frequent in their research in 2009 [46]. Thus we could get a conclusion that maybe c.729_730insTT is a hotspot mutation in Chinese Patients [47].

In our patients, the c.729_730insTT variant, detected in $16.78 \%$ of the MUT cases, was the very common variant. The other frequently observed variants were c. $1106 \mathrm{G}>\mathrm{A}, \mathrm{c} .914 \mathrm{~T}>\mathrm{C}$ and c.323G $>\mathrm{A}$, with each variant accounting for $7.69 \%, 6.99 \%, 6.29 \%$, respectively. This reflects that c.729_730insTT and c.323G > A are frequent variants in Chinese population.

A total of 34 variants were identified among our $158 \mathrm{cblC}$ defect pedigrees, including 2 novel variants, namely the c.89G >A and c.415_416delinsTA variant, each of them are nonsense variant, yielding a truncated protein at residue 30 and 139, respectively, resulting in incomplete protein activity. The the softwares' prediction are all deleterious, however, further research should be performed to confirm their pathogenecity..

In the 60 different variants we have identified among 70 MUT type patients, 41 of them were previously reported and 16 of these variants were novel, including one 5'UTR variant (c.-39-2A > G), 1 splicing mutations (c. $1560+2 T>C$ ), 1 small deletion (c.1233_1235delCAT), 3 deletions of single nucleotide (c.1102delG, c.1645delC and c.1787delA), 3 insertions of single nucleotide

Page 15/20 
(c.445_446insA, c.1107dupT and c.1581_1582insA), 1 nonsense (c.7A $>$ T) and 6 missense mutations (c.422C > A, c.652C > G, c. $893 \mathrm{~T}>\mathrm{A}, \mathrm{c} .1330 \mathrm{~A}>\mathrm{T}$ c. $1673 \mathrm{C}>\mathrm{T}, \mathrm{c} .1678 \mathrm{~T}>\mathrm{C}$ ), most of which have been found only once and are restricted to one single family.

Methylmalonyl-CoA mutase (MCM) is encoded by MUT gene encoding 750 amino acids, containing 2 important main functional domains:1)an N-terminal ( $\beta / \alpha) 8$ barrel domain (residues $86-423)$ that accommodates the substrate and 2$)$ a $C$-terminal $(\alpha / \beta) 5$ cofactor (adenosylcobalamin)-binding domain (residues 578-750). The two main domains are connected by a long linker region (residues $424-577)$ that could enclose the $(\beta / \alpha) 8$ barrel domain $[9,48]$.

In our study, 6 novel frameshift variants and one nonsense variants were detected, leading to introduction of a subsequent premature termination codon and production of truncated protein. The novel splicing variant is predicted to affect splicing, and the stability of mRNA, and 5'UTR variant would affect the level of translation [49] . One deletion of 3 nucleotides causes Isoleucine deletion at redisue 410, which could affect the binding of substrate. The remaining 9 novel missense variants were identified in 9 different patients. Seven of them are located in the $\mathrm{N}$-terminal $(\beta / \alpha) 8$ barrel domain, while 2 others (c.1673C $>T$ and c.1678T >C) are located in the linker domain. The former would influence the binding of substrate, and the latter may affect the binding of substrates, enzymes and cobalamin. The novel missense mutations, being likely disease-causing, in 9 different pedigrees all occurred in segments highly conserved across the tested species, suggesting that these residues are crucial for stability and function of MCM.

One patient had the c.826-2A > G variant in SUCLG1 gene in a homozygous state, which had been reported to be harmful [50]. Another pedigree with diseased proband, the parents both harboured the c.981-2A > TA heterozygous variant in $L M B R D 1$ gene. Some Database indicated that this variant is binign (Illumina Clinical Services Laboratory), another one literature showed that its' pathogenicity is uncertain [51]. However, the Mutationtaster predicted that it's disease-causing, the specific function needs further experimental study.

For all the novel variants, Mutation taster, Polymorphism phenotyping (PolyPhen) and SIFT were calculated, and the results show that they are all disease causing. However, further research should be done to verify our speculation of the pathogenicity.

For all genes, sequence analysis is performed first, followed by deletion or duplication analysis if only one pathogenic or no variant has been detected. Here in our study real-time quantitative PCR (Q-PCR) technology was used to test deletions/duplications in the MMACHC/ MUT genes. After Q-PCR, only 2 pedigrees have found exon 13 deletion in MUT gene [52]. The exon 13 deletion, was confirmed by microarray-based comparative genomic hybridization [31].

According to the current station, early detection and treatment are critical strategies to improve the outcome of MMA patients, however, varying degrees of psychoneurotic sequel would lower the quality of their life. Thus preimplantation genetic diagnosis or screening (PGD/PGS) are the optimal effective prevention ways at present, which would be a prevailing trend to help those affected with MMA in the development of MMA prenatal diagnosis, although it costs a lot.

\section{Conclusion}

Novel variants were identified in $M M A C H C$ and $M U T$ gene, which would broaden the spectrum of these two genes, especially for Chinese Population. Rasing awareness for MMA has the potential to improve the patients' outcome by timely initiation of targeted treatment. Prenatal genetic diagnosis is an accurate and feasible method to help prevent the delivery of MMA patients.

\section{Abbreviations}

cblC: cobalamin(cbl) C PCR: polymerase chain reaction MMA: Methylmalonic acidemia or aciduria MCM: methylmalonyl coenzyme A mutase GC-MS: gas chromatography-mass spectrometry FPLA: Fluorescence polarization immunoassay PGD/PGS: preimplantation genetic diagnosis or screening

\section{Declarations}

Ethics approval and consent to participate: 
This study was approved by the Medical Ethics Committee in the First Affiliated Hospital of Zhengzhou University. All of the analysed samples were obtained with signed informed consent.

\section{Consent for publication:}

All of the analysed samples were obtained with signed informed consent.

\section{Availability of data and material:}

All data generated or analysed during this study are included in this published article and its supplementary information files.

\section{Competing interests:}

The authors declare that they have no competing interests

\section{Funding:}

This work was supported by grants from the Hospital Internal Funding from the First Affiliated Hospital of Zhengzhou University.

\section{Authors' contributions:}

XK and SH conceived the study, SH and LL summarized all the data and involved in histological analysis, ZJ and XK provided expertise for data interpretation and suggestions for manuscript preparation. SH wrote the manuscript.

\section{Acknowledgements:}

Not applicable

\section{References}

1. Chen M, Zhuang J, Yang J, Wang D, Yang Q. Atypical hemolytic uremic syndrome induced by CbIC subtype of methylmalonic academia: A case report and literature review. Medicine 2017; 96(43):e8284.

2. Zhou X, Cui Y, Han J. Methylmalonic acidemia: Current status and research priorities. Intractable \& rare diseases research 2018; 7(2):73-8.

3. Fowler B, Leonard JV, Baumgartner MR. Causes of and diagnostic approach to methylmalonic acidurias. Journal of inherited metabolic disease 2008; 31(3):350-60.

4. Baumgartner MR, Horster F, Dionisi-Vici C, Haliloglu G, Karall D, Chapman KA, Huemer M, Hochuli M, Assoun M, Ballhausen D et al. Proposed guidelines for the diagnosis and management of methylmalonic and propionic acidemia. Orphanet journal of rare diseases 2014; 9:130.

5. Fraser JL, Venditti CP. Methylmalonic and propionic acidemias: clinical management update. Current opinion in pediatrics 2016; 28(6):682-93.

6. Carrillo-Carrasco N, Chandler RJ, Venditti CP. Combined methylmalonic acidemia and homocystinuria, cblC type. I. Clinical presentations, diagnosis and management. J Inherit Metab Dis 2012; 35(1):91-102.

7. Carrillo-Carrasco N, Venditti CP. Combined methylmalonic acidemia and homocystinuria, cblC type. II. Complications, pathophysiology, and outcomes. Journal of inherited metabolic disease 2012; 35(1):103-14.

8. Lin Y, Lin C, Lin W, Zheng Z, Han M, Fu Q. Mild clinical features of isolated methylmalonic acidemia associated with a novel variant in the MMAA gene in two Chinese siblings. BMC medical genetics 2018; 19(1):114.

9. Martinez MA, Rincon A, Desviat LR, Merinero B, Ugarte M, Perez B. Genetic analysis of three genes causing isolated methylmalonic acidemia: identification of 21 novel allelic variants. Molecular genetics and metabolism 2005; 84(4):317-25.

10. Coelho D, Suormala T, Stucki M, Lerner-Ellis JP, Rosenblatt DS, Newbold RF, Baumgartner MR, Fowler B. Gene identification for the cbID defect of vitamin B12 metabolism. The New England journal of medicine 2008; 358(14):1454-64. 
11. Yu HC, Sloan JL, Scharer G, Brebner A, Quintana AM, Achilly NP, Manoli I, Coughlin CR, 2nd, Geiger EA, Schneck U et al. An Xlinked cobalamin disorder caused by mutations in transcriptional coregulator HCFC1. American journal of human genetics 2013; 93(3):506-14.

12. Keyfi F, Talebi S, Varasteh AR. Methylmalonic Acidemia Diagnosis by Laboratory Methods. Reports of biochemistry \& molecular biology 2016; 5(1):1-14.

13. Zong Y, Liu N, Zhao Z, Kong X. Prenatal diagnosis using genetic sequencing and identification of a novel mutation in MMACHC. BMC Med Genet 2015; 16:48.

14. Maalej M, Tej A, Bouguila J, Tilouche S, Majdoub S, Khabou B, Tabbebi M, Felhi R, Ammar M, Mkaouar-Rebai E et al. Clinical, Molecular, and Computational Analysis in two cases with mitochondrial encephalomyopathy associated with SUCLG1 mutation in a consanguineous family. Biochemical and biophysical research communications 2018; $495(2): 1730-7$.

15. Levtova A, Waters PJ, Buhas D, Levesque S, Auray-Blais C, Clarke JTR, Laframboise R, Maranda B, Mitchell GA, Brunel-Guitton C et al. Combined malonic and methylmalonic aciduria due to ACSF3 mutations: benign clinical course in an unselected cohort. Journal of inherited metabolic disease 2018.

16. Abily-Donval L, Torre S, Samson A, Sudrie-Arnaud B, Acquaviva C, Guerrot AM, Benoist JF, Marret S, Bekri S, Tebani A. Methylmalonyl-CoA Epimerase Deficiency Mimicking Propionic Aciduria. International journal of molecular sciences 2017; 18(11).

17. Hu S, Mei S, Liu N, Kong X. Molecular genetic characterization of cblC defects in 126 pedigrees and prenatal genetic diagnosis of pedigrees with combined methylmalonic aciduria and homocystinuria. BMC medical genetics 2018; $19(1): 154$.

18. Liu YP, Ma YY, Wu TF, Wang Q, Li XY, Ding Y, Song JQ, Huang Y, Yang YL. [Abnormal findings during newborn period of 160 patients with early-onset methylmalonic aciduria]. Zhonghua er ke za zhi = Chinese journal of pediatrics 2012; 50(6):410-4.

19. Wang F, Han LS, Hu YH, Yang YL, Ye J, Qiu WJ, Zhang YF, Gao XL, Wang Y, Gu XF. [Analysis of gene mutations in Chinese patients with methylmalonic acidemia and homocysteinemia]. Zhonghua er ke za zhi = Chinese journal of pediatrics 2009; 47(3):189-93.

20. Han LS, Huang Z, Han F, Ye J, Qiu WJ, Zhang HW, Wang Y, Gong ZW, Gu XF. Clinical features and MUT gene mutation spectrum in Chinese patients with isolated methylmalonic acidemia: identification of ten novel allelic variants. World journal of pediatrics : WJP2015; 11(4):358-65.

21. Manoli I, Sloan JL, Venditti CP: Isolated Methylmalonic Acidemia. In: GeneReviews((R)). Edited by Adam MP, Ardinger HH, Pagon RA, Wallace SE, Bean LJH, Stephens K, Amemiya A. Seattle (WA); 1993.

22. Kruszka PS, Manoli I, Sloan JL, Kopp JB, Venditti CP. Renal growth in isolated methylmalonic acidemia. Genet Med 2013; 15(12):990-6.

23. Kang L, Liu Y, Shen M, Liu Y, He R, Song J, Jin Y, Li M, Zhang Y, Dong H et al. A study on a cohort of 301 Chinese patients with isolated methylmalonic acidemia. J Inherit Metab Dis 2020; 43(3):409-23.

24. Rosenblatt DS, Aspler AL, Shevell MI, Pletcher BA, Fenton WA, Seashore MR. Clinical heterogeneity and prognosis in combined methylmalonic aciduria and homocystinuria (cblC). J Inherit Metab Dis 1997; 20(4):528-38.

25. Weisfeld-Adams JD, Bender HA, Miley-Akerstedt A, Frempong T, Schrager NL, Patel K, Naidich TP, Stein V, Spat J, Towns S et al. Neurologic and neurodevelopmental phenotypes in young children with early-treated combined methylmalonic acidemia and homocystinuria, cobalamin C type. Mol Genet Metab 2013; 110(3):241-7.

26. Han B, Cao Z, Tian L, Zou H, Yang L, Zhu W, Liu Y. Clinical presentation, gene analysis and outcomes in young patients with early-treated combined methylmalonic acidemia and homocysteinemia (cblC type) in Shandong province, China. Brain \& development 2016; 38(5):491-7.

27. Scolamiero E, Cozzolino C, Albano L, Ansalone A, Caterino M, Corbo G, di Girolamo MG, Di Stefano C, Durante A, Franzese G et al. Targeted metabolomics in the expanded newborn screening for inborn errors of metabolism. Molecular bioSystems 2015; 11(6):1525-35.

28. Zhang Y, Yang YL, Hasegawa Y, Yamaguchi S, Shi CY, Song JQ, Sayami S, Liu P, Yan R, Dong JH et al. Prenatal diagnosis of methylmalonic aciduria by analysis of organic acids and total homocysteine in amniotic fluid. Chinese medical journal 2008; $121(3): 216-9$. 
29. Inoue $\mathrm{Y}$, Ohse M. Prenatal diagnosis of methylmalonic aciduria by measuring methylmalonic acid in dried amniotic fluid on filter paper using gas chromatography-mass spectrometry. Analytical and bioanalytical chemistry 2011; 400(7):1953-8.

30. Lerner-Ellis JP, Tirone JC, Pawelek PD, Dore C, Atkinson JL, Watkins D, Morel CF, Fujiwara TM, Moras E, Hosack AR et al. Identification of the gene responsible for methylmalonic aciduria and homocystinuria, cblC type. Nature genetics 2006; 38(1):93-100.

31. Wang J, Zhan H, Li FY, Pursley AN, Schmitt ES, Wong LJ. Targeted array CGH as a valuable molecular diagnostic approach: experience in the diagnosis of mitochondrial and metabolic disorders. Molecular genetics and metabolism 2012; 106(2):22130.

32. Hu S, Mei S, Bai Y, Kong X. [Analysis of MUT gene mutations and prenatal diagnosis for 20 pedigrees affected with isolated methylmalonic aciduria]. Zhonghua yi xue yi chuan xue za zhi = Zhonghua yixue yichuanxue zazhi = Chinese journal of medical genetics 2018; 35(4):471-4.

33. Chang JT, Chen YY, Liu TT, Liu MY, Chiu PC. Combined methylmalonic aciduria and homocystinuria cblC type of a Taiwanese infant with c.609G>A and C.567dupT mutations in the MMACHC gene. Pediatrics and neonatology 2011; 52(4):223-6.

34. Forny P, Froese DS, Suormala T, Yue WW, Baumgartner MR. Functional characterization and categorization of missense mutations that cause methylmalonyl-CoA mutase (MUT) deficiency. Human mutation 2014; 35(12):1449-58.

35. Huemer M, Scholl-Burgi S, Hadaya K, Kern I, Beer R, Seppi K, Fowler B, Baumgartner MR, Karall D. Three new cases of late-onset cblC defect and review of the literature illustrating when to consider inborn errors of metabolism beyond infancy. Orphanet journal of rare diseases 2014; 9:161.

36. Nogueira C, Aiello C, Cerone R, Martins E, Caruso U, Moroni I, Rizzo C, Diogo L, Leao E, Kok F et al. Spectrum of MMACHC mutations in Italian and Portuguese patients with combined methylmalonic aciduria and homocystinuria, cblC type. Molecular genetics and metabolism 2008; 93(4):475-80.

37. Lerner-Ellis JP, Anastasio N, Liu J, Coelho D, Suormala T, Stucki M, Loewy AD, Gurd S, Grundberg E, Morel CF et al. Spectrum of mutations in MMACHC, allelic expression, and evidence for genotype-phenotype correlations. Human mutation 2009; 30(7):1072-81.

38. Yuen YP, Lai CK, Chan YW, Lam CW, Tong SF, Chan KY. DNA-based diagnosis of methylmalonic aciduria and homocystinuria, cblC type in a Chinese patient presenting with mild developmental delay. Clinica chimica acta; international journal of clinical chemistry 2007; 375(1-2):171-2.

39. Liu MY, Yang YL, Chang YC, Chiang SH, Lin SP, Han LS, Qi Y, Hsiao KJ, Liu TT. Mutation spectrum of MMACHC in Chinese patients with combined methylmalonic aciduria and homocystinuria. Journal of human genetics 2010; 55(9):621-6.

40. Imtiaz F, Al-Mubarak BM, Al-Mostafa A, Al-Hamed M, Allam R, Al-Hassnan Z, Al-Owain M, Al-Zaidan H, Rahbeeni Z, Qari A et al. Spectrum of Mutations in 60 Saudi Patients with Mut Methylmalonic Acidemia. JIMD reports 2016; 29:39-46.

41. Kumari C, Kapoor S, Varughese B, Pollipali SK, Ramji S. Mutation Analyses in Selected Exons of the MUT Gene in Indian Patients with Methylmalonic Acidemia. Indian journal of clinical biochemistry : IJCB 2017; 32(3):266-74.

42. Worgan LC, Niles K, Tirone JC, Hofmann A, Verner A, Sammak A, Kucic T, Lepage P, Rosenblatt DS. Spectrum of mutations in mut methylmalonic acidemia and identification of a common Hispanic mutation and haplotype. Human mutation 2006; 27(1):31-43.

43. Acquaviva C, Benoist JF, Callebaut I, Guffon N, Ogier de Baulny H, Touati G, Aydin A, Porquet D, Elion J. N219Y, a new frequent mutation among mut(degree) forms of methylmalonic acidemia in Caucasian patients. European journal of human genetics : EJHG 2001; 9(8):577-82.

44. Ogasawara M, Matsubara Y, Mikami H, Narisawa K. Identification of two novel mutations in the methylmalonyl-CoA mutase gene with decreased levels of mutant mRNA in methylmalonic acidemia. Human molecular genetics 1994; 3(6):867-72.

45. Liu MY, Liu TT, Yang YL, Chang YC, Fan YL, Lee SF, Teng YT, Chiang SH, Niu DM, Lin SJ et al. Mutation Profile of the MUT Gene in Chinese Methylmalonic Aciduria Patients. JIMD reports 2012; 6:55-64.

46. Wang F, Han L, Ye J, Qiu W, Zhang Y, Gao X, Wang Y, Yang Y, Gu X. [Analysis of the MUT gene mutations in patients with methylmalonic acidemia]. Zhonghua yi xue yi chuan xue za zhi = Zhonghua yixue yichuanxue zazhi = Chinese journal of medical genetics 2009; 26(5):485-9. 
47. Chen Z, Zhang X, Huang J, Wen P, Wang G, Tang G, Zu Y, Chen X, Cui D, Zhang M et al. [Analysis of MUT gene mutations in a patient with isolated methylmalonic acidemia]. Zhonghua yi xue yi chuan xue za zhi = Zhonghua yixue yichuanxue zazhi = Chinese journal of medical genetics 2015; 32(2):218-21.

48. Acquaviva C, Benoist JF, Pereira S, Callebaut I, Koskas T, Porquet D, Elion J. Molecular basis of methylmalonyl-CoA mutase apoenzyme defect in 40 European patients affected by mut(o) and mut- forms of methylmalonic acidemia: identification of 29 novel mutations in the MUT gene. Human mutation 2005; 25(2):167-76.

49. Sample PJ, Wang B, Reid DW, Presnyak V, McFadyen IJ, Morris DR, Seelig G. Human 5 ' UTR design and variant effect prediction from a massively parallel translation assay. Nat Biotechnol 2019; 37(7):803-9.

50. Liu YP, Li XY, Ding Y, Wang Q, Song JQ, Zhang Y, Li DX, Qin YP, Yang YL. [Clinical and laboratory studies on four Chinese patients with succinate-CoA ligase deficiency noticed by mild methylmalonic aciduria]. Zhonghua er ke za zhi = Chinese journal of pediatrics 2016; 54(5):365-9.

51. Rutsch F, Gailus S, Miousse IR, Suormala T, Sagne C, Toliat MR, Nurnberg G, Wittkampf T, Buers I, Sharifi A et al. Identification of a putative lysosomal cobalamin exporter altered in the cblF defect of vitamin B12 metabolism. Nature genetics 2009; 41(2):234-9.

52. Ginzinger DG. Gene quantification using real-time quantitative PCR: an emerging technology hits the mainstream. Experimental hematology 2002; 30(6):503-12. 